ISBN 978-93-84422-76-9

International Conference on Research \& Innovation in Environment, Civil and Architecture Engineering

(RIECAE-17)

Bangkok (Thailand), Feb. 6-7, 2017

\title{
Open-Window Active Noise Control for Environmental Noise
}

\author{
Wongeun Oh \\ Sunchon National University, Korea. Email id: owg@scnu.ac.kr
}

\begin{abstract}
The single channel duct-type open-window active noise control(ANC) systems have several advantages than the traditional ANC windows because of the simplicity and no inter-channel interference. We examine the effects of the single channel ANC window on environmental noise when the main noise source is the human voice. The experiments using a scale model room are conducted, and the results show that the single channel open-window ANC can reduce the energy of average speech spectrum.
\end{abstract}

Keywords: Active Noise Control, Open-Window ANC, Environmental noise, Openness.

\section{Introduction}

The continuous exposure to urban noise has been proved to be harmful to human by an increasing number of health studies. The urban noise exists not only on the road side but in the buildings or houses. Since most of the in-room noise was transmitted through open windows, the noise can be effectively reduced by closing windows. However, this solution is less effective when the residents want to natural air or openness.

This problem might be solved with the ANC (Active Noise Control) window technology. The ANC system generates an anti-noise with the opposite phase to cancel the undesired noise. Today, the ANC system is recognized as one of the effective technique to reduce low frequency noise [1].

Many ANC window systems have been proposed to reduce the noise transmission through a window [2]. However, despite the successful results, some practical problem still prevents to the real applications. They have the computational complexity and the performance degradation by the interference between channels. A single channel duct-type open-window ANC system is suggested to overcome the problems and proved to be effective for reducing the transmitted noise even when the window is open [3]-[5].

In this paper, we examine the effects of the duct-type single channel ANC window when the primary noise source is the human voice. The human voice can be the main noise source where the street-side buildings, the offices in the mall, and the houses near parks, etc. The scale model room experiment shows the window reduces the speech energy and affects the speech intelligibility.

\section{Single-Channel Duct-Type Open-Window ANC}

A cross section sketch of the duct-type open-window ANC installed in a room is shown in Fig. 1 [5]. 


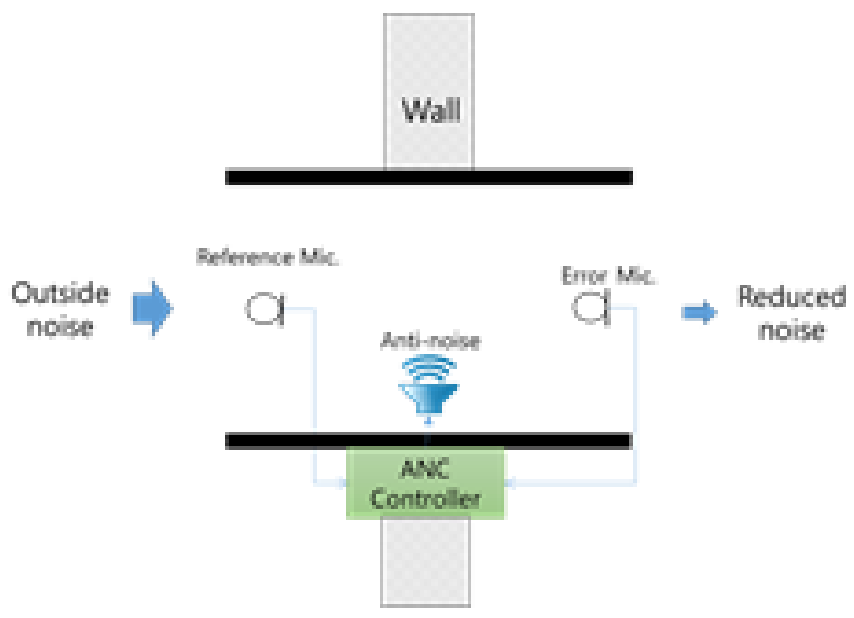

Fig. 1 Duct-type open-window ANC system

The system consists of a short length duct, a reference microphone, an error microphone, a control loudspeaker, and an adaptive controller. The reference microphone captures the exterior noise and sends it to the adaptive controller. Then the controller calculates 180 degrees out of phase signal, and the loudspeaker plays it. This makes the destructive interference between the noise and the loudspeaker sound. Therefore, the exterior noise energy passing through the window can be decreased.

\section{Experiments and Results}

The block diagram of the experimental setup is shown in Fig. 2. The dimension of the model room is $0.6 \mathrm{~m}$ x $1.2 \mathrm{~m} \mathrm{x} 0.75 \mathrm{~m}$ and the walls are covered with $2.5 \mathrm{~cm}$ thick sound absorbing materials. The dimension of the window is $0.12 \mathrm{~m} \times 0.19 \mathrm{~m}$, and the duct type active window with length $0.52 \mathrm{~m}$ is installed on it. And the 'Demo S-Fan 90' and related software tools by Silentium [6] are used as an open-window ANC controller.

The pre-recorded outside noise is played by Yamaha MSP5 monitoring loudspeaker, and an Audix TR40A microphone is used for measuring noise in the room. The EASERA [7] software is used for the signal generation and the data analysis.

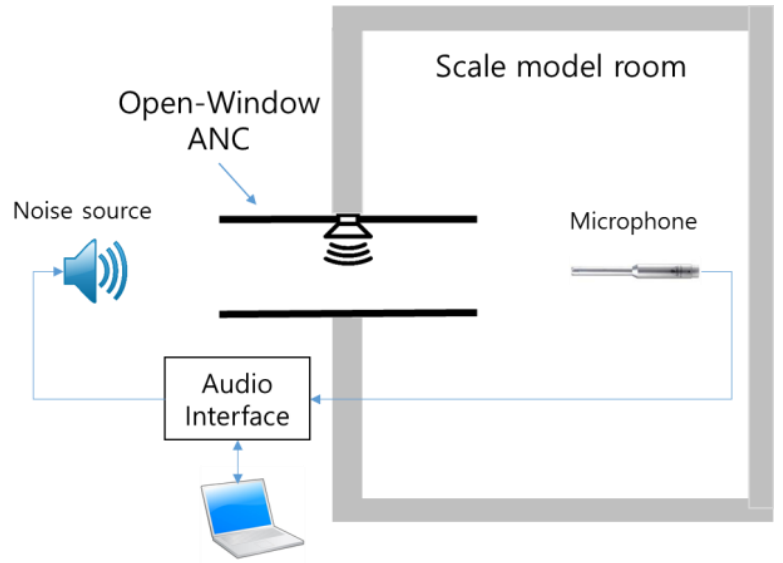

Fig. 2 Experimental setup of the duct-type ANC window

\subsection{Frequency response of the open-window ANC}

Fig. 3 is the magnitude frequency response of the open window with and without ANC. The result shows that the outside noise is reduced approximately $5 \mathrm{~dB}$ between $400 \mathrm{~Hz}-1800 \mathrm{~Hz}$ frequency range. The range is important because most of the LTASS (Long-term average speech spectrum) energy lies below $1 \mathrm{kHz}$ with a peak at $500 \mathrm{~Hz}$ and gradually decreases. This means that the duct-type open-window ANC system can reduce effectively the outside noise caused by the human voice. 


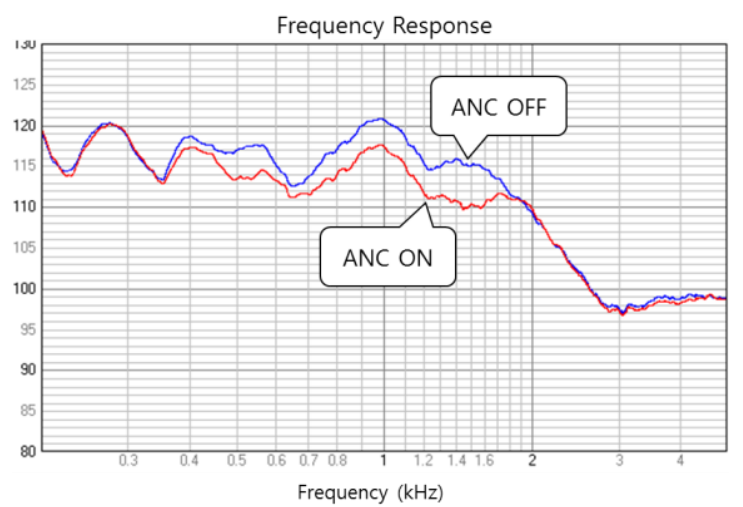

Fig. 3 Frequency response when ANC On and ANC Off

\subsection{Effect on the speech intelligibility}

The STI (Speech Transmission Index) was used to measure the effect of the ANC on human speech. The STI is most frequently used intelligibility criteria in room acoustics [8]. The measured values are 0.831 when the ANC is off and 0.784 when the ANC is on. The intelligibility degraded approximately 0.047 because the ANC cancels voice energy transmitted through the window.

\section{Conclusion}

The duct-type open-window ANC system for the noise from human voice was examined using a scale model room. The frequency response of ANC was reduced approximately $5 \mathrm{~dB}$ between $400 \mathrm{~Hz}-1800 \mathrm{~Hz}$ frequency range in which most of LTASS energy lies. And the STI was degraded about 5\% when ANC was activated.

\section{Acknowledgements}

This research was supported by Basic Science Research Program through the National Research Foundation of Korea(NRF) funded by the Ministry of Education (NRF-2014R1A1A2057077).

\section{References}

[1] S. M. Kuo, K. Kuo, and Woon Seng Gan, "Active noise control: Open problems and challenges," 2010 Int. Conf. Green Circuits Syst., no. 1, pp. 164-169, 2010.

[2] B. Lam and W.-S. Gan, “Active Acoustic Windows: Towards a Quieter Home," IEEE Potentials, vol. 35, no. 1, pp. 11-18, 2016.

[3] W. Oh, "Performance Evaluation of Active Windows Using Speech Intelligibility," in International Conference on Engineering Technologies and Big Data Analytics (ETBDA'2016), 2016, pp. 66-68.

[4] W. Oh, "An Experimental Study on the Simple Active Noise Control Windows," in 3rd International conference on Innovative Engineering Technologies (ICIET'2016), 2016, pp. 87-90.

[5] W. Oh, "Noise reduction in rooms using a single channel active noise controller," in Engineering \& Technology, Computer, Basic \& Applied Sciences, 2016.

[6] Silentium LTD., "Silentium's Demo S-Fan 90.” [Online]. Available: http://www.silentium.com/products/demo-s-fan90/.

[7] AFMG, "EASERA : Electronic and Acoustic System Evaluation and Response Analysis." [Online]. Available: http://easera.afmg.eu/.

[8] H. J. M. Steeneken and T. Houtgast, "Basics of the STI -measuring method," pp. 22-24, 2002. 